\title{
Integrated profiling identifies SLC5A6 and MFAP2 as novel diagnostic and prognostic biomarkers in gastric cancer patients
}

\author{
TAO SUN, DANHUA WANG, YING PING, YIWEN SANG, YIBEI DAI, YIYUN WANG, \\ ZHENPING LIU, XIUZHI DUAN, ZHIHUA TAO and WEIWEI LIU \\ Department of Laboratory Medicine, The Second Affiliated Hospital of Zhejiang \\ University School of Medicine, Hangzhou, Zhejiang 310009, P.R. China
}

Received April 30, 2019; Accepted September 2, 2019

DOI: 10.3892/ijo.2019.4944

\begin{abstract}
Gastric cancer (GC) is one of the leading causes of malignancy-associated mortality worldwide. However, the underlying molecular mechanisms of GC are unclear and the prognosis of GC is poor. Therefore, it is important and urgent to explore the underlying mechanisms and screen for novel diagnostic and prognostic biomarkers, as well as therapeutic targets. In the current study, scale-free gene co-expression networks were constructed using weighted gene co-expression network analysis, the potential associations between gene sets and clinical features were investigated, and the hub genes were identified. The gene expression profiles of GSE38749 were downloaded from the Gene Expression Omnibus database. RNA-seq and clinical data for GC from The Cancer Genome Atlas were utilized for verification. Furthermore, the expression of candidate biomarkers in gastric tissues was investigated. Survival analysis was performed using Kaplan-Meier and log-rank test. The predictive role of candidate biomarkers in GC was evaluated using a receiver operator characteristic (ROC) curve. Gene Ontology, gene set enrichment analysis and gene set
\end{abstract}

Correspondence to: Professor Zhihua Tao or Dr Weiwei Liu, Department of Laboratory Medicine, The Second Affiliated Hospital of Zhejiang University School of Medicine, 88 Jiefang Road, Hangzhou, Zhejiang 310009, P.R. China

E-mail: zrtzh@zju.edu.cn

E-mail: liuweiwei@zju.edu.cn

Abbreviations: $\mathrm{BP}$, biological process; CC, cellular component; DEG, differentially expressed gene; GC, gastric cancer; GEO, Gene Expression Omnibus; GO, Gene Ontology; GS, gene significance; KEGG, Kyoto Encyclopedia of Genes and Genomes; MFAP2, microfibril-associated protein 2; ME, module eigengene; MF, molecular function; MM, module membership; OS, overall survival; WGCNA, weighted genes co-expression network analysis; TCGA, The Cancer Genome Atlas; TOM, topological overlap measure; ROC, receiver operating characteristic

Key words: gastric cancer, weighted gene co-expression network analysis, TCGA, candidate gene, overall survival, prognosis, receiver operating characteristic variation analysis methods were used to interpret the function of candidate biomarkers in GC. A total of 29 modules were identified via the average linkage hierarchical clustering. A significant module consisting of 48 genes associated with clinical traits was found; three genes with high connectivity in the clinical significant module were identified as hub genes. Among them, SLC5A6 and microfibril-associated protein 2 (MFAP2) were negatively associated with the overall survival, and their expression was elevated in GC compared with non-tumor tissues. Additionally, ROC curves indicated that SLC5A6 and MFAP2 showed a good diagnostic power in discriminating cancerous from normal tissues. SLC5A6 and MFAP2 were identified as novel diagnostic and prognostic biomarkers in GC patients; both of these genes were first reported here in connection with GC and deserved further research.

\section{Introduction}

Gastric cancer (GC) represents the most common malignant tumors of the digestive tract (1). Despite significant improvements in diagnostic and therapeutic strategies of GC over the past decades, the incidence and mortality rates of GC are still increasing due to lack of early diagnostic tactics and effective treatments (1). However, if GC patients are diagnosed and treated early, either by endoscopy or surgery, the 5-year survival rate could exceed $90 \%$ (2). Consequently, prompt diagnosis of GC is significantly improving prognosis. Therefore, it is extremely important to discover novel candidate genes, which play important roles in the initiation and development of GC, and help to reduce mortality rates and improve prognosis.

Thanks to the continuous innovation of technologies on microarray and high-throughput sequencing, an increasing number of biomarkers and therapeutic targets have been identified and applied in clinic (3-6), particularly in the field of medical oncology (7-9). The Cancer Genome Atlas (TCGA) is a large database, which provides publicly available genomic and clinical information for various cancer types (10). Using this database, researchers can comprehensively and accurately study the biology and pathology of each cancer (11). Additionally, TCGA contributes to precise cancer diagnosis and individualized treatment through the identification of novel candidate genes and clinical information linked to cancer (12). 
Co-expression analysis is a powerful strategy to construct scale-free gene co-expression networks (13). The weighted gene co-expression network analysis (WGCNA) is widely used to analyze large-scale data sets and find modules of highly correlated genes (14). In addition, WGCNA has been successfully utilized to investigate associations between gene sets and clinical traits, and to identify potential candidate biomarkers of various cancer types (15-18), including prostate (15), esophageal (17) and cervical cancer (18). Thus, WGCNA provides a functional interpretation tool for cancer biology and has brought new insights into understanding the molecular pathogenesis and prognostics of cancer.

In order to explore the underlying mechanisms and to identify novel prognostic biomarkers and therapeutic targets of GC, in the present study, WGCNA was performed using microarray data of GC patients downloaded from the Gene Expression Omnibus (GEO) database, and significant modules and genes were identified. These genes were further confirmed using TCGA datasets and may act as oncogenes. Taken together, SLC5A6 and microfibril-associated protein 2 (MFAP2) were identified as novel diagnostic and prognostic biomarkers, which may provide new insights into early diagnosis and targeted therapy for patients with GC.

\section{Materials and methods}

Preparing gene expression profile data and clinical information. The workflow of this study is presented in Fig. S1. The gene expression profile data and clinical information for GC were downloaded from the GEO database; GSE38749 (https://www. ncbi.nlm.nih. gov/geo/query/acc.cgi?acc=GSE38749) is gene expression data based on the GPL570 platform (Affymetrix Human Genome U133 plus 2.0 Array) (19). The GSE38749 dataset includes 15 gastric cancer samples. Tumor staging was conducted as per the criteria detailed in the 8th edition of the TNM Staging Manual of the American Joint Committee on Cancer (20). The gene expression levels downloaded with the database were calculated as fragments per kilo base of transcript per million mapped reads. Clinical data contained age, gender, TNM stage and survival time. Clustering analysis was performed by calculating the correlation coefficient matrix, which indicated that the genes were qualified for subsequent analyses and that variation was small. Data standardization was performed using the robust multi-array average algorithm in the affy package within Bioconductor (http://www.bioconductor.org) in R 3.5.2 (https://cran.rstudio.com/).

Screening for differentially expressed genes (DEGs). Limma package was used to screen the differentially expressed genes with R 3.5.2 (https://cran.rstudio.com/). According to an established statistical method (21), 21,648 genes were identified for further studies. Based on the variance of each gene in each sample, the genes with standard deviations $>0$ were selected as DEGs. The top 50\% of variant genes based on an analysis of variance $(10,824$ genes) were selected for WGCNA.

Constructing the co-expression network. WGCNA is a systematic biological method for constructing scale-free networks using gene expression data. All analyses were performed using the WGCNA v1.68 package in R 3.5.2 (https://cran.rstudio. com/). Firstly, the similarity matrix of gene expression was constructed by calculating the Pearson's correlation coefficient between two genes. Then, the gene expression similarity matrix was converted into the adjacency matrix and the network type is assigned; $\beta=12$ was selected as soft threshold. The purpose of this step was the strengthening of strong and weakening of weak correlations at the expression level. Then, the adjacency matrix was transformed into the topological matrix (TOM); TOM was used to describe the degree of association between genes. Based on TOM, which represented diverse degree of genes, (1-TOM) was used for hierarchical clustering of genes. The dynamic tree cut algorithm was used to module recognition and the most representative gene in each module was called eigenvector gene or module eigengene (ME), representing the overall level of gene expression in this module and the first principal component in each module.

Identifying clinically significant modules. Two approaches were used to identify a correlation between modules and clinical information obtained from patients with GC. Analyses were performed using the WGCNA v1.68 and Cor packages in R 3.5.2 (https://cran.rstudio.com/). The minimum number of genes per module was 30 , the correlation threshold of hub genes was 0.90 and the unsigned network edge threshold was 0.05. Firstly, the expression profiles of a gene in all samples and of a vector gene were calculated using Pearson's correlation as module membership (MM). ME was defined as the first principal component of each gene module and the expression of ME was considered representative of all genes in a given module. Clinically significant modules were identified by calculating the correlation between ME and clinical traits, and the degree of the connection was measured. Gene significance (GS) was used to measure this degree; a higher GS indicated increased biological significance of genes. MS were defined as the mean GS of all the genes involved in the module.

Enrichment analysis of Gene Ontology $(G O)$ and Kyoto Encyclopedia of Genes and Genomes (KEGG) pathways. DAVID 6.8 (https://david.ncifcrf.gov/) was used for GO and KEGG enrichment analysis. GO and KEGG pathway enrichment analyses were used to identify potentially involved biological processes, molecular function and cellular component. GO contained three categories: i) Biological processes (BPs); ii) cellular components (CCs); and iii) molecular function (MF). The potential biological features and pathways of genes in the modules were further explored using DAVID (https://david.ncifcrf.gov/). To avoid missing discoveries, the significance threshold was adjusted based on an established statistical method (22); data were evaluated with the adjusted cut-off value $\mathrm{P}<0.1$. The associations between genes in 'black' module were visualized with Cytoscape 3.5.1 (https://cytoscape.org/), as this module had the highest correlation coefficient with clinical traits.

Identifying and validating hub genes. The correlation of genes was calculated using absolute of Pearson's correlation values via Cor package in R 3.5.2 (https://cran.rstudio.com/). Genes that had high correlation with a module were regarded as hub genes of this module (cor. MM, >0.9). Furthermore, 

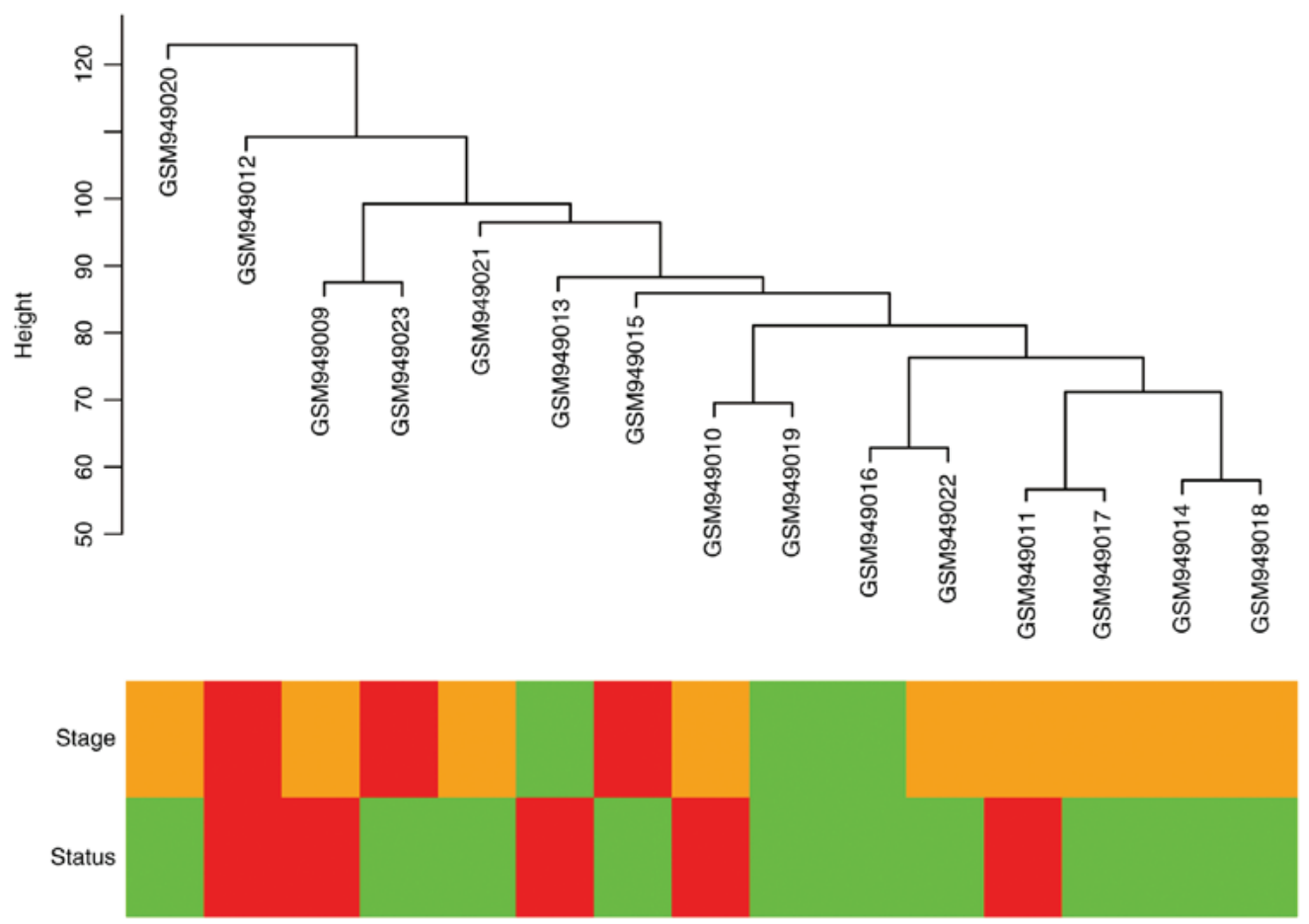

Figure 1. Cluster dendrogram for 15 gastric cancer samples from the GSE38749 dataset. Classification is according to American Joint Committee on Cancer; with stage reported as stage III, green; stage IIIa, yellow; stage IIIb, red; and status reported as survival, red; and death, green.

to validate the hub genes, the data downloaded from TCGA (https://cancergenome.nih.gov/) contained clinical information and RNA sequencing data. mRNA sequencing data were normalized using edgeR package in $\mathrm{R} 3.5 .2$ (https://cran. rstudio.com/). GC data from TCGA contained 406 samples, which included 375 tumor and 31 corresponding adjacent normal tissues. Hub genes were validated in 375 tumor tissue samples using WGCNA. Kaplan Meier-plotter 3.0.0 (www. kmplot.com) was used for the survival analysis of hub genes.

Immunohistochemistry analysis. The Human Protein Atlas (https://www.proteinatlas.org/) was used to validate candidate hub genes via immunohistochemistry. Images were obtained from the following sources: i) SLC5A6 in normal tissue ( $\mathrm{n}=6$; https://www.proteinatlas.org/ ENSG00000138074-SLC5A6/tissue/stomach); ii) SLC5A6 in tumor tissue $(\mathrm{n}=12$; https://www.proteinatlas.org/ ENSG00000138074-SLC5A6/pathology/tissue/stomach+cancer\# ihc); iii) MFAP2 in normal tissue, (n=5; https://www.proteinatlas.org/ENSG00000117122-MFAP2/tissue/stomach); and iv) MFAP2 in tumor tissue ( $\mathrm{n}=12$; https://www.proteinatlas. org/ENSG00000117122-MFAP2/pathology/stomach+cancer). The immunohistochemical staining pattern of each tissue sample was annotated manually. Images of sections were evaluated and scored by two pathologists independently. The annotation was based on staining intensity (negative, weak, moderate or strong) and fraction of stained cells $(<25 \%, 25 \sim 75 \%,>75 \%)$. The staining quantity of each protein via IHC was determined as the percentage of stained cells in 10 high power fields. All annotation data and immunohistochemistry images from the standard tissue set of 44 tissues, together with data from extended tissue samples analyzed in the present investigation and all antibody validation data are publicly available at v18.proteinatlas.org.
Statistical analysis. Data are presented as the mean \pm SEM and were analyzed with SPSS (version 19.0; IBM Corp.). Significant differences were calculated using one-way ANOVA with Dunnett's or Newman-Keuls test, or by two-tailed Student's t-test. $\mathrm{P}<0.05$ was considered to indicate a statistically significant difference.

\section{Results}

WGCNA construction and identification of clinically significant modules. Cluster analysis was performed on the samples of GSE38749 using average linkage and Pearson's correlation (Fig. 1). The co-expression network was constructed using co-expression analysis. To ensure a scale-free network, the power $\beta=12$ was identified as soft-threshold in the present study (Fig. 2). A total of 29 modules were identified via the average linkage hierarchical clustering, calculating with MEs and combing adjacent modules with the same module and height $=0.25$ (Fig. 3A). As shown in Fig. 3B and C, the 'black' module ( $\mathrm{r}=0.73$; $\mathrm{P}=0.002$ ) was found to have the highest association with cancer prognosis. Therefore, this module was selected as the key clinically significant module for subsequent analysis. The modules 'skyblue' $(\mathrm{R}=0.70 ; \mathrm{P}=0.0034)$ and 'blue' $(\mathrm{R}=0.71 ; \mathrm{P}=0.0031)$ also had high correlations with clinical traits and further evaluation may focus on the correlation between genes and the disease. The connectivity of integrated modules and genes with clinical traits was calculated and the correlation was significantly different $\left(\mathrm{R}=0.64 ; \mathrm{P}=9.7 \times 10^{-7}\right.$; Fig. 3D). In addition, the correlation of modules was calculated according to MEs (Fig. 4).

GO and enrichment analysis of the key module. The genes in the key clinically significant module were categorized into three functional groups, namely BP, CC and MF. The key 

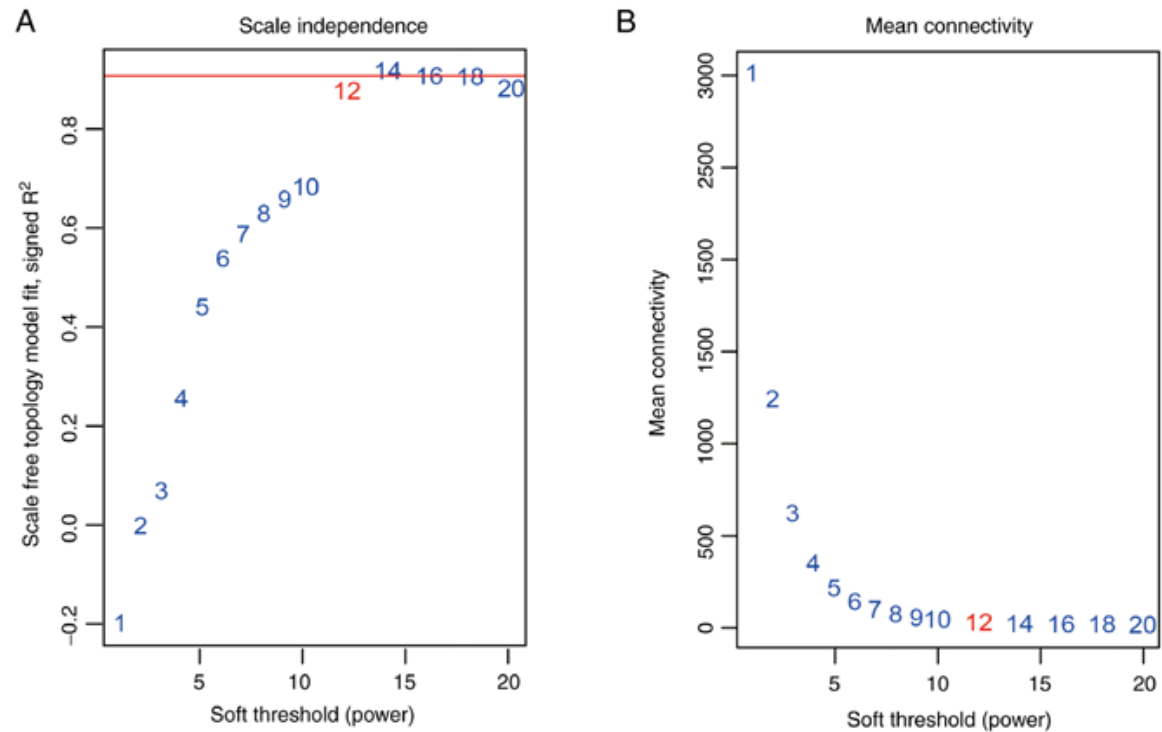

Figure 2. Determination of the soft-threshold in weighted genes co-expression network analysis. (A) Analysis of the scale-free fit index for various soft-thresholds determining scale independence. (B) Analysis of the mean connectivity for various soft-thresholds.
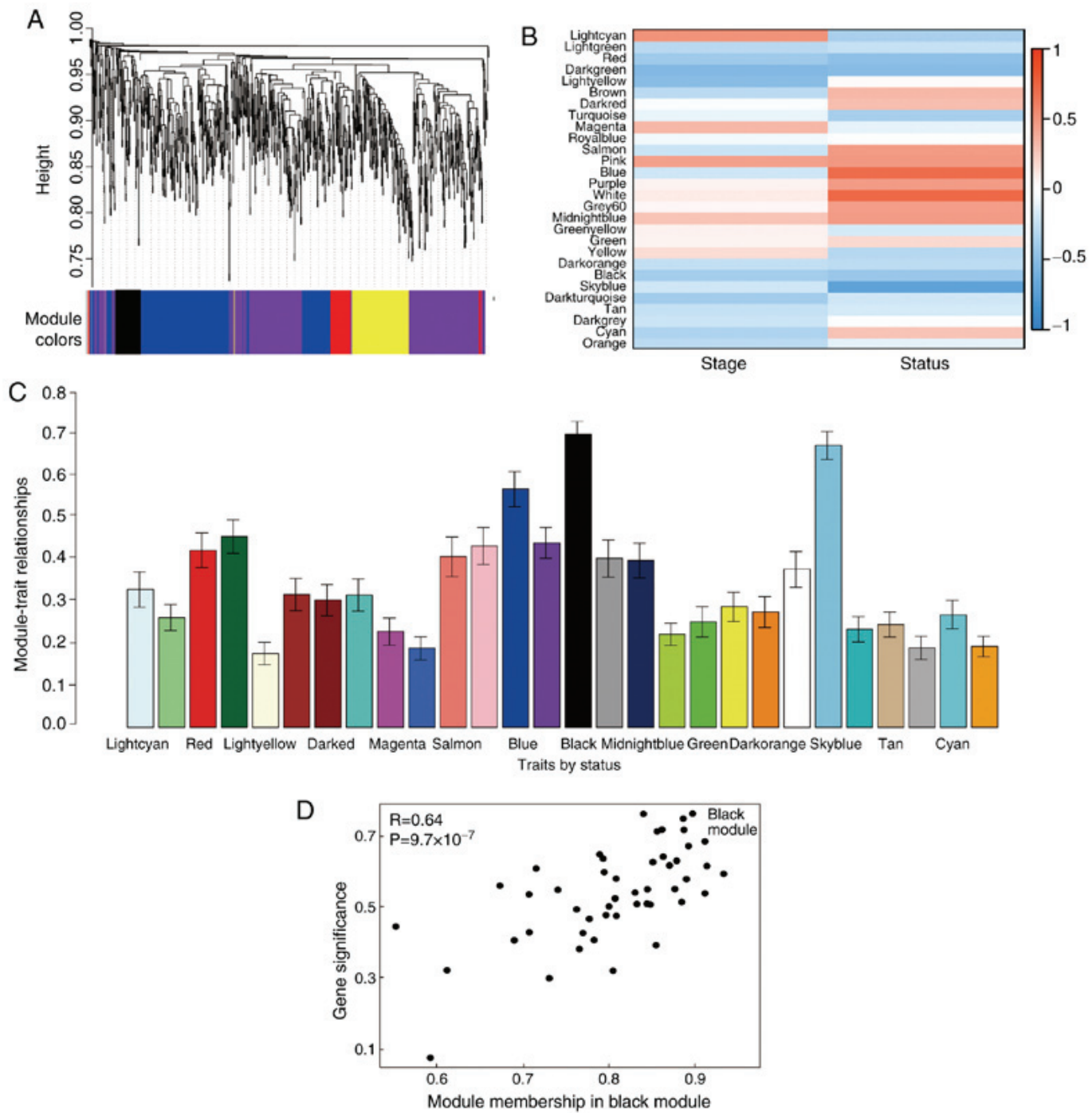

Figure 3. Identification of modules associated with the clinical traits of gastric cancer. (A) Cluster dendrogram of all differentially expressed genes clustered on a dissimilarity measure. (B) Heatmap of the correlation between module eigengenes and clinical traits of gastric cancer. (C) Distribution of average gene significance errors in the modules associated with tumor prognosis of gastric cancer. (D) Connectivity of gene significance with module membership in the 'black' module. 

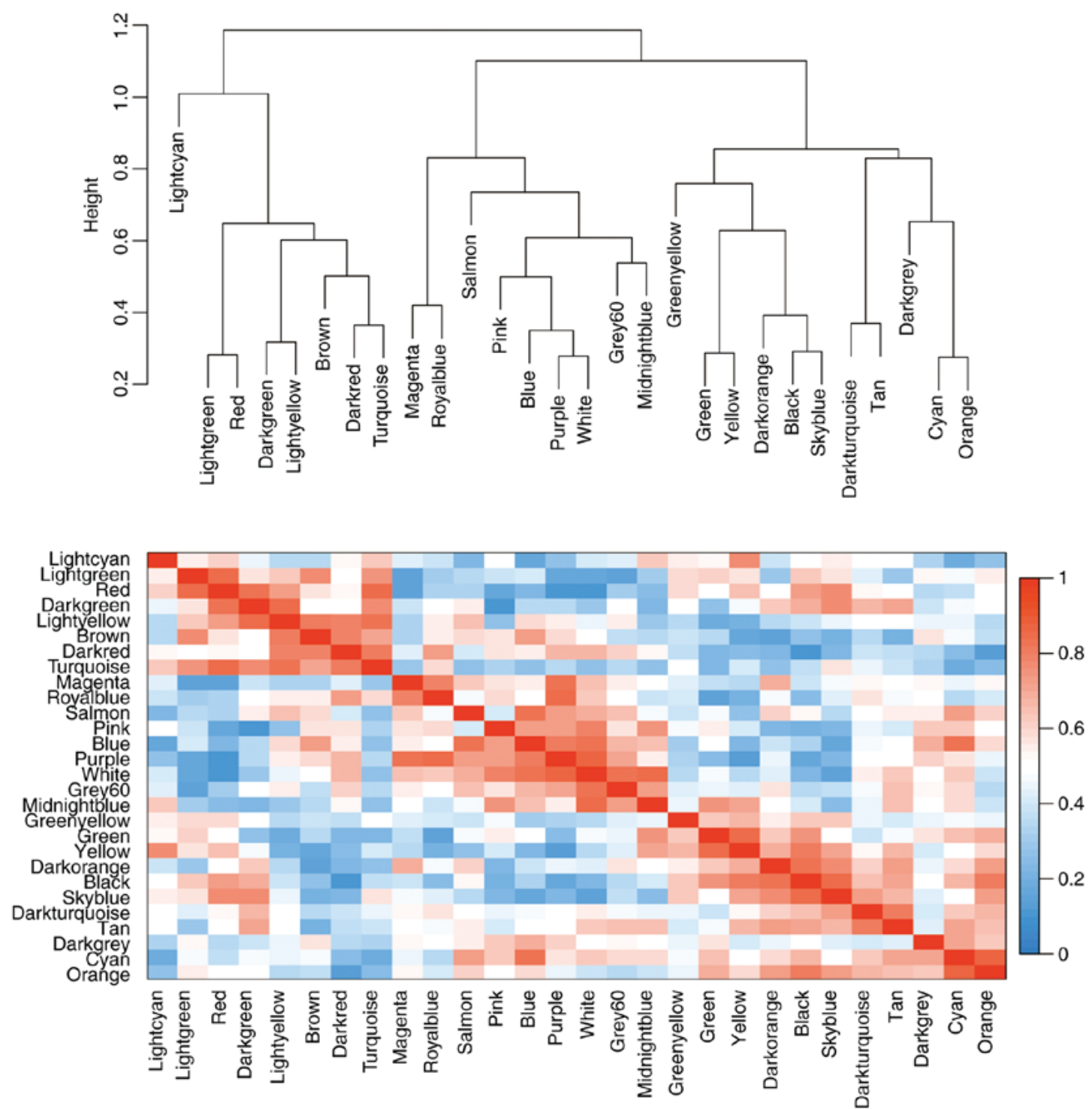

Figure 4. Correlation of modules based on their eigengenes. Visualization of hierarchical clustering dendrogram of the eigengenes (top); the eigengene network represents the relationships among modules. Heat map of the eigengene adjacency (bottom); the colored bars on the left and below indicate the module for each row or column.

module genes in the BP group were associated with positive regulation of endothelial cell proliferation, lung development, positive regulation of peptidyl-threonine phosphorylation, cellular response to fibroblast growth factor stimulus, collagen fibril organization, DNA repair, DNA duplex unwinding, transmembrane transport, cellular response to amino acid stimulus and cellular response to transforming growth factor $\beta$-stimulus (Fig. 5A). In the MF groups in metallopeptidase activity, telomeric DNA binding and identical protein binding associated genes were enriched and in the $\mathrm{CC}$ group proteinaceous extracellular matrix genes were enriched (Fig. 5A). KEGG pathway analysis demonstrated that these genes were associated with positive regulation of endothelial cell proliferation, proteinaceous extracellular matrix and metallopeptidase activity (Fig. 5B). The gene co-expression network was constructed including all genes from the 'black' module using Cytoscape, SLC5A6 and MFAP2 were most closely associated with the prognosis of GC in the 'black' module and were selected as hub genes for further analysis (Fig. 5C).
Identification of clinically significant hub genes. Based on the cut-off criteria (cor. MM, $>0.9$ ), three genes with high connectivity in the key module were identified $(\mathrm{P}<0.001$; Fig. 6). SLC5A6 and MFAP2 were significantly negatively associated

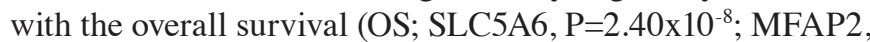
$\left.\mathrm{P}=8.00 \times 10^{-8}\right)$ and EVA1A showed a non-significant negative association with OS ( $\mathrm{P}=0.16$; Fig. 7).

Validation of clinically significant hub genes. Using TCGA data, no correlation between cancer stage and SLC5A6 or MFAP2 was identified (Fig. 8A and B). RNA-sequencing expression levels of SLC5A6 or MFAP2 were significantly increased in tumor tissues compared with normal tissues $(\mathrm{P}<0.01$; Fig. $8 \mathrm{C}$ and $\mathrm{D})$.

SLC5A6 and MFAP2 may serve as novel diagnostic markers for $G C$. ROC curves showed that SLC5A6 and MFAP2 exhibited excellent diagnostic efficiency for GC $(\mathrm{P}<0.001$; Fig. 9). The area under the ROC curve was calculated using 375 tumor 


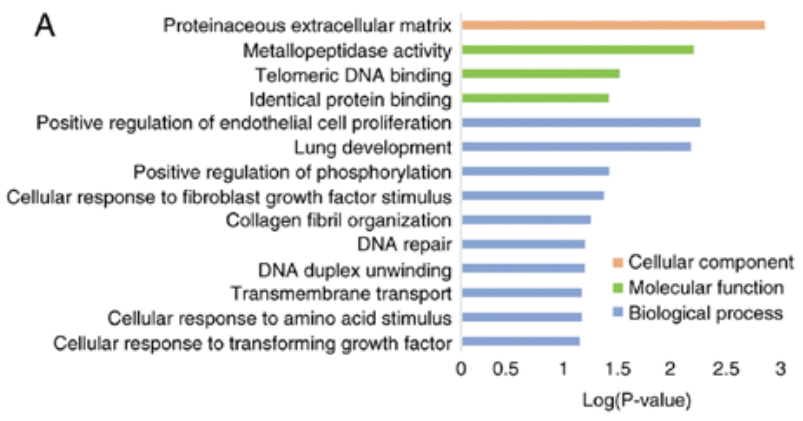

B
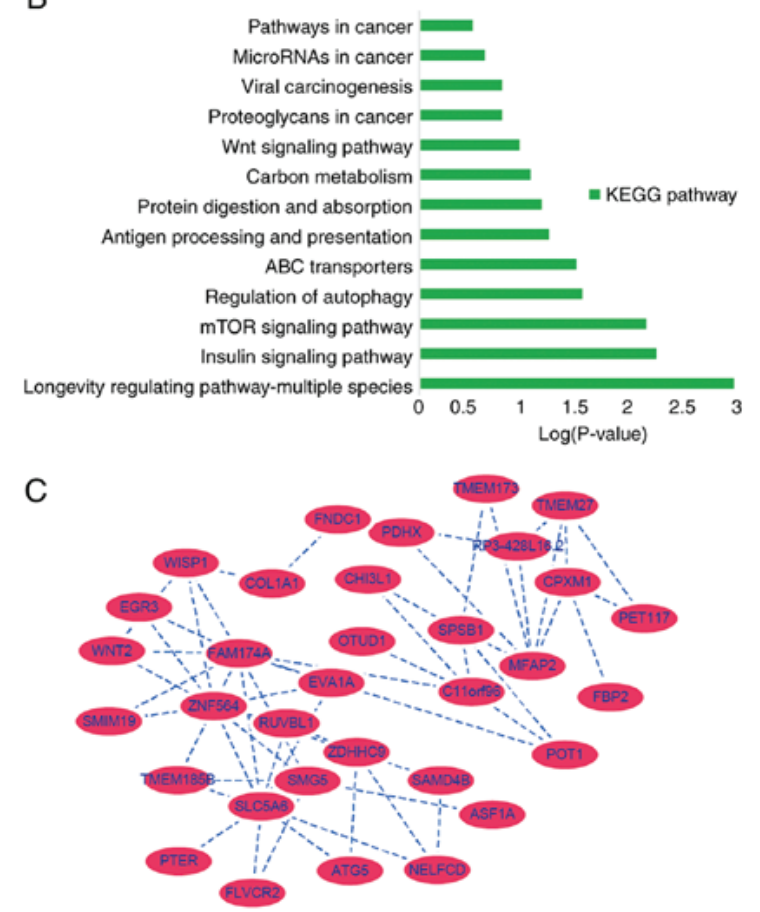

Figure 5. Pathway analysis of hub genes. Significantly enriched genes using Gene Ontology annotations for the 'black' module, including (A) biological processes, molecular function and cellular components and (B) Kyoto Encyclopedia of Genes and Genome pathways. (C) Weighted co-expression network for the 'black' module genes, with nodes displayed according to the connectivity of genes.

and 32 normal samples; for SLC5A6 an area under the curve of 1.000 and for MFAP2 0.9842 were determined. These results indicated that SLC5A6 and MFAP2 may serve as novel diagnostic marker for GC.

Furthermore, the diagnostic efficiency of SLC5A6 and MFAP2 was verified via immunohistochemistry results using the Human Protein Atlas database. Data for the two hub genes were obtained, including antibody staining, intensity and quantity in immunohistochemistry. Immunohistochemistry results indicated that the protein expression of SLC5A6 in tumor tissues was increased compared with normal tissues (Fig. 10). However, protein expression of MFAP2 in tumor and normal tissues was not markedly different (Fig. 11). This was inconsistent with the results of mRNA expression using the TCGA data.

\section{Discussion}

GC has high reoccurrence even after combined treatments and it is a serious health thread (23). Multiple factors are involved in the tumorigenesis and progression of $\mathrm{GC}$, including tumor suppressor gene inactivation, oncogene overexpression, tumor microenvironment remodeling, lifestyle, environmental factors and others $(1,24)$. Due to the roles of genetic factors in GC occurrence, development, progression and prognosis, microarray and high-throughput sequencing can further help to study the function of genes at the whole genome level (25). As a systematic biology method to depict how clinical characteristics associate with genes, in this study, WGCNA was applied to investigate co-expression in GC and normal tissues.

In the present study, 21,648 DEGs were identified and further processed and the top $50 \%$ of genes (10,824 genes) were selected for WGCNA analysis. Using comprehensive analyses of GS, MS and MM, it was inferred that SLC5A6, MFAP2 and EVA1A were the hub genes of the 'black' module. However, only SLC5A6 and MFAP2 were identified as clinically significant hub genes, which were further successfully validated using TCGA data. Additionally, SLC5A6 and MFAP2 exhibited excellent diagnostic efficiency for GC tissues compared with normal tissues.

According to the distribution of mean GS and errors in the modules associated with prognosis of GC, the 'black' module was identified as the clinical significant module, and the majority of enriched genes were associated with BP, including the positive regulation of endothelial cell proliferation. KEGG pathway analysis indicated that these genes were involved in longevity regulating pathway-multiple species, insulin signaling pathway and mTOR signaling pathway. These results implied that the cluster of genes in this significant module may play an important role in promoting tumor growth, increasing proteinaceous extracellular matrix production and activating metallopeptidase. The abnormal proliferation of endothelial cells is closely associated with occurrence and development of GC $(26,27)$. In addition, metalloproteinases activation and tumor microenvironment remodeling pave the way for cancer metastasis and progress (28-30). Further studies may explore the roles and functions of the hub genes identified in this study.

SLC5A6 and MFAP2 were negatively associated with OS of GC patients. mRNA expression of these genes were significantly increased in tumor tissues compared with normal tissues; however, it did not significantly vary by tumor stage. This suggested that these two genes have only a small impact on disease grading. The protein expression of SLC5A6 was significantly increased in tumor tissues, but protein expression of MFAP2 was observed in both in normal and tumor tissues. According to these results, it was suggested that SLC5A6 and MFAP2 were novel diagnostic and prognostic biomarkers for GC, with SLC5A6 potentially being more reliable than MFAP2.

MFAP2 is an abundant component of microfibrils $(31,32)$. The research of MFAP2 mainly focuses on its role in regulating the deposition of proelastin on microfibers to form elastic fibers (31), and few studies have been conducted to investigate the function of MFAP2 in cancer. MFAP2 has been identified to be co-expressed in association with the $\mathrm{NF}-\kappa \mathrm{B} / \mathrm{Snail} / \mathrm{Y} Y 1 / \mathrm{RKIP}$ signaling pathway in multiple myeloma (33). In addition, MFAP2 has been shown to be markedly elevated in head and neck squamous cell carcinoma, particularly in lymph node metastasis (34). Recently, Wang et al (35) demonstrated that MFAP2 can 

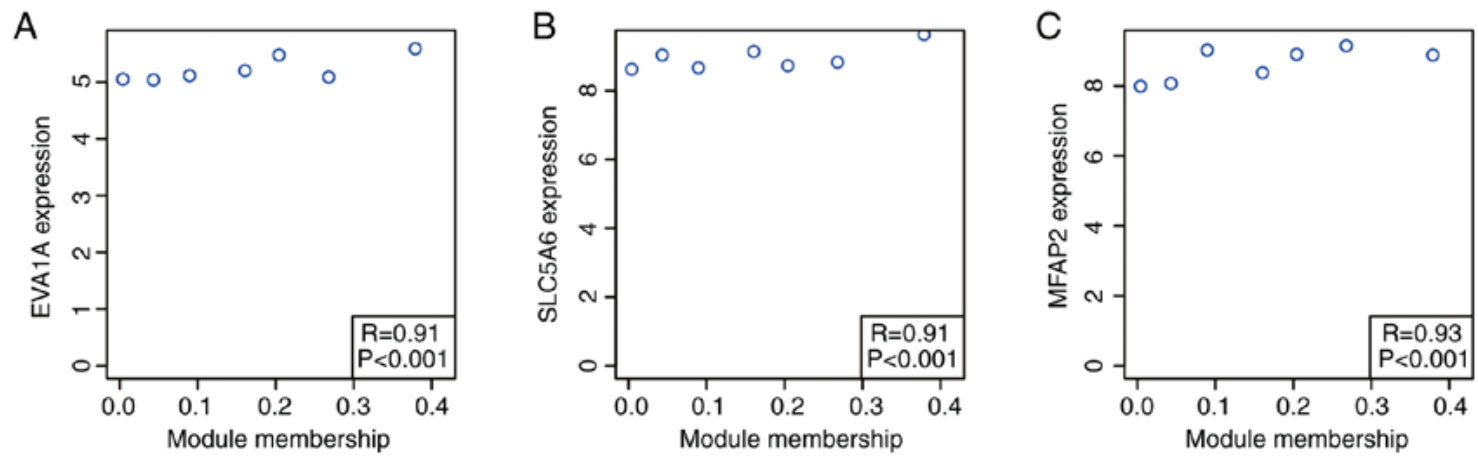

Figure 6. Correlation of three genes with module membership in the 'black' module. Associations between module membership for the 'black' module and the expression of (A) EVA1A, (B) SLC5A6 and (C) MFAP2 are plotted. MFAP2, microfibril-associated protein 2.
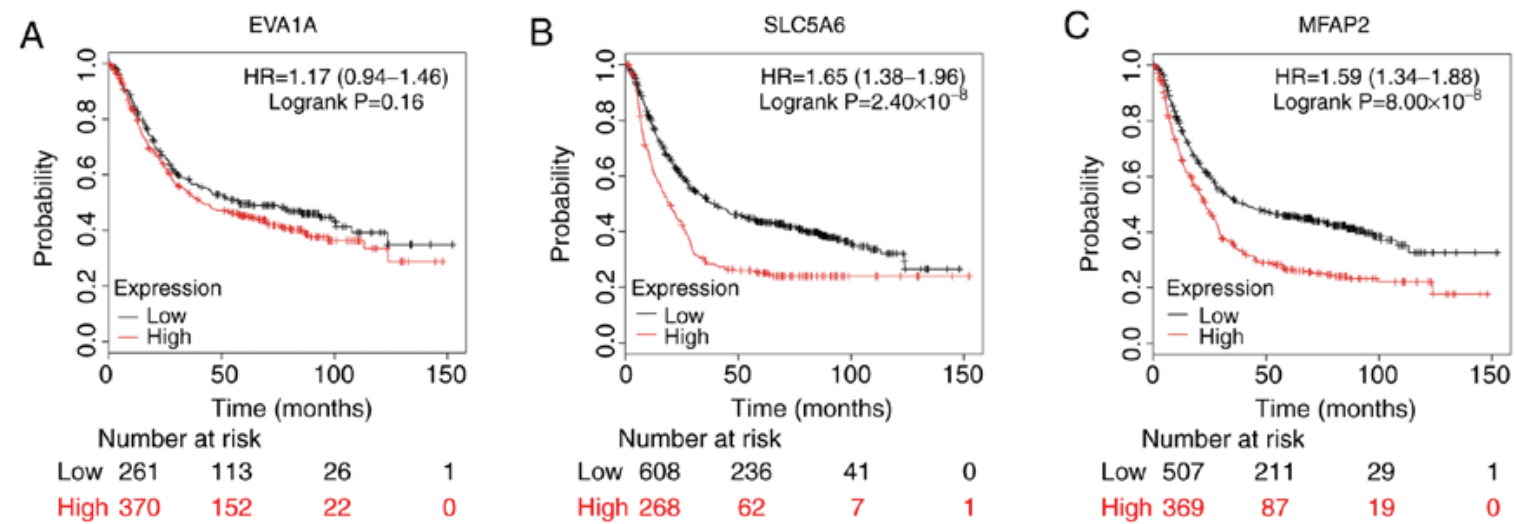

Figure 7. Overall survival of patients with gastric cancer associated with hub gene expression. Curves were created using the Kaplan Meier-plotter and patients were stratified into high- and low-level expression groups according to median expression. Survival is plotted for (A) EVA1A, (B) SLC5A6 and (C) MFAP2 expression. MFAP2, microfibril-associated protein 2.
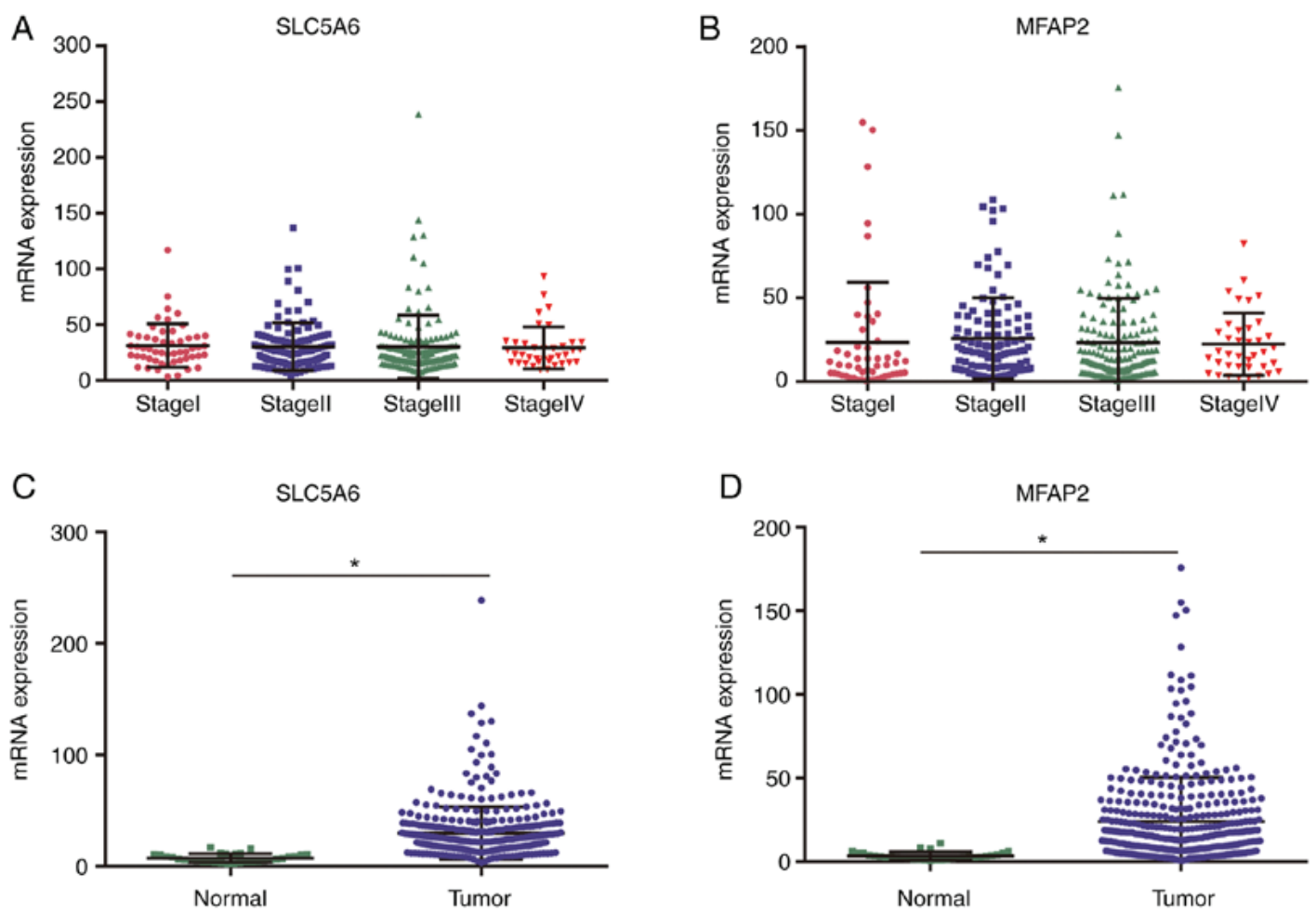

Figure 8. Validation of SLC5A6 and MFAP2 expression in gastric cancer. mRNA expression at different stages for (A) SLC5A6 and (B) MFAP2, with P>0.01 for all comparisons (ANOVA). mRNA expression in tumor and normal tissues for (C) SLC5A6 and (D) MFAP2. "P<0.0001 (paired Student's t-test). MFAP2, microfibril-associated protein 2. 

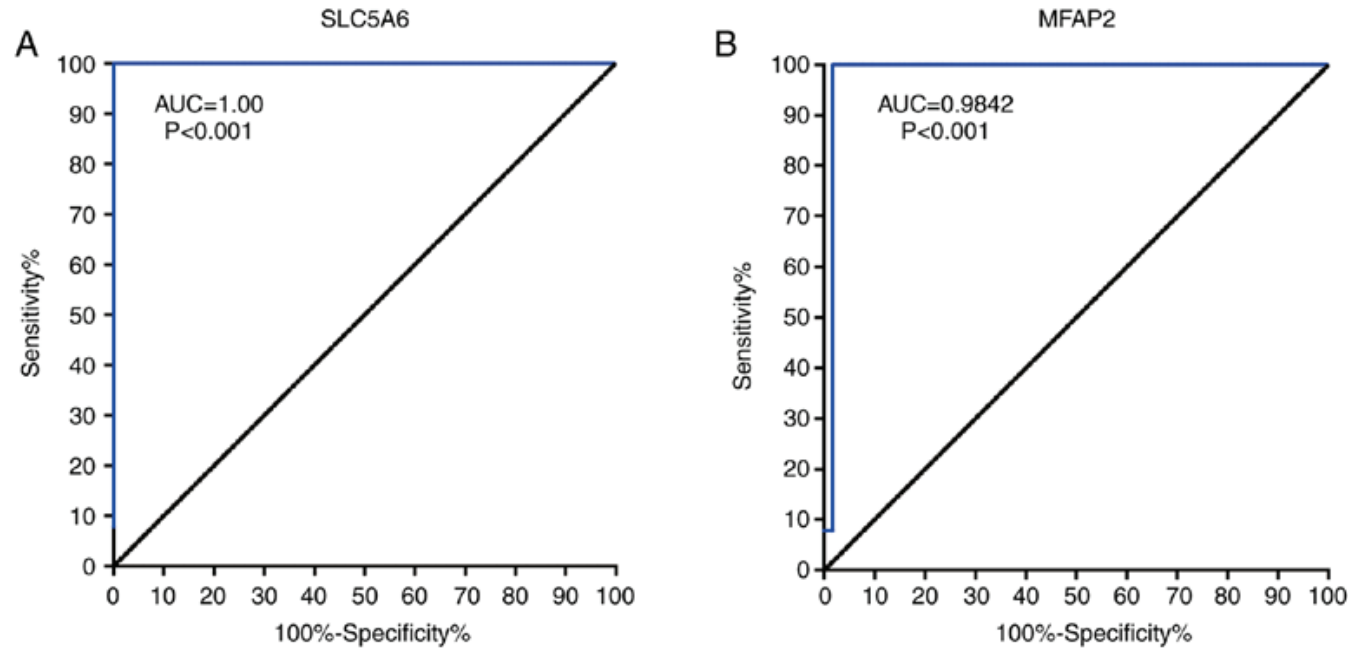

Figure 9. ROC curves for hub genes. Area under the ROC curve were determined for (A) SLC5A6 and (B) MFAP2. ROC, receiver operating characteristic; AUC, area under the curve; MFAP2, microfibril-associated protein 2.
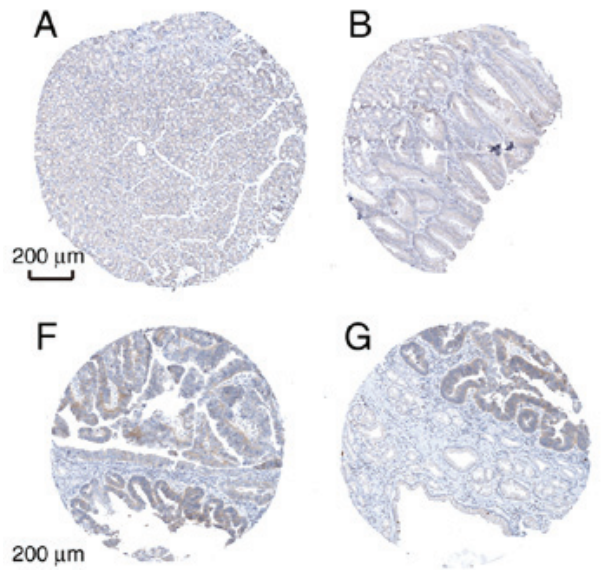
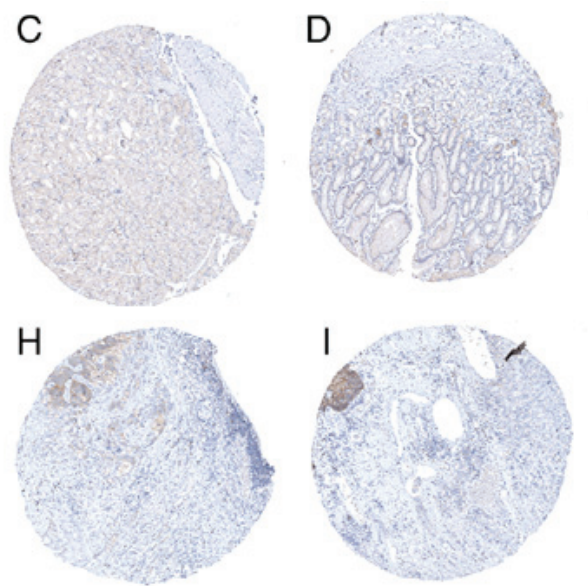
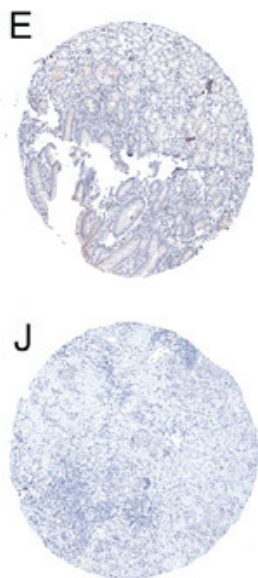

Figure 10. Immunohistochemistry analysis of SLC5A6 expression in gastric cancer samples. Data were obtained from the Human Protein Atlas. (A-E) Protein levels of SLC5A6 in normal tissue (staining: not detected; intensity: negative; quantity: negative). (F) Protein levels of SLC5A6 in tumor tissue (staining: medium; intensity: moderate; quantity: 25\% 75\%). (G) Protein levels of SLC5A6 in tumor tissue (staining: medium; intensity: moderate; quantity: 25 75\%). (H) Protein levels of SLC5A6 in tumor tissue (staining: not detected; intensity: weak; quantity: <25\%). (I) Protein levels of SLC5A6 in tumor tissue (staining: not detected; intensity: weak; quantity: $<25 \%$ ). (J) Protein levels of SLC5A6 in tumor tissue (staining: not detected; intensity: weak; quantity: $<25 \%$ ). Scale bar, $200 \mu \mathrm{m}$. Normal tissue ( $\mathrm{n}=6)$, tumor tissue $(\mathrm{n}=12)$.
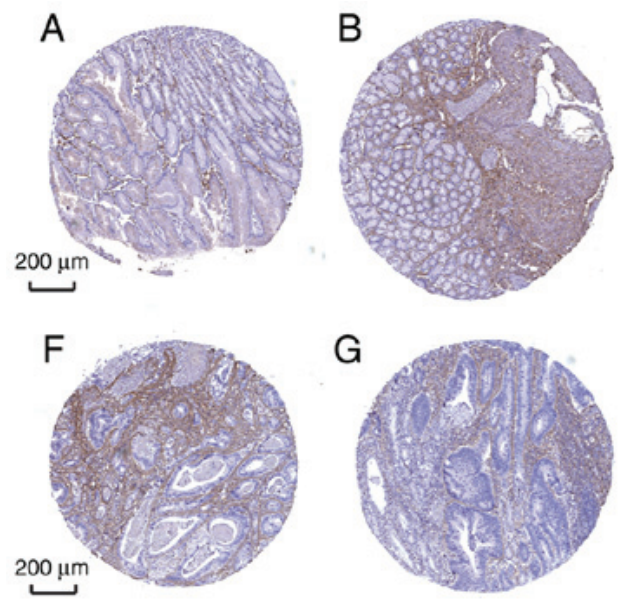
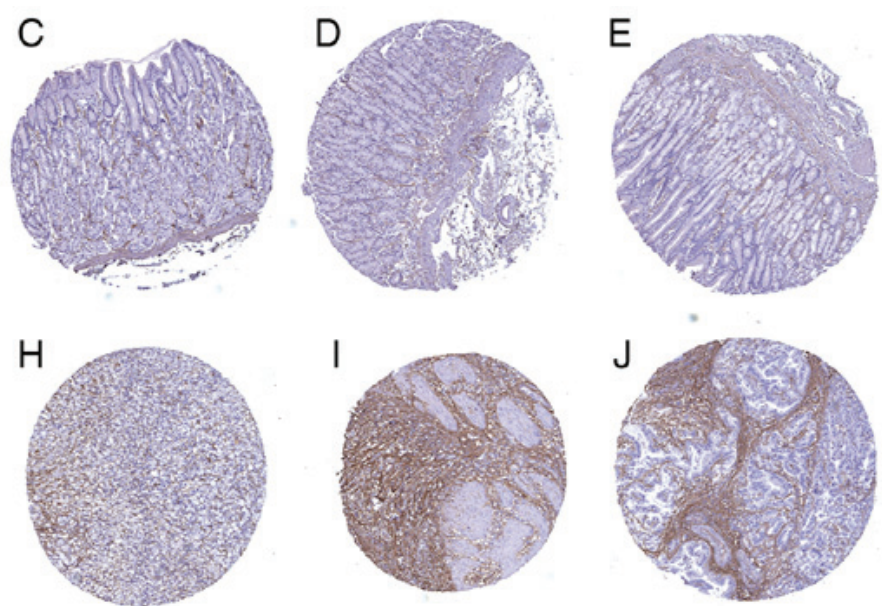

Figure 11. Immunohistochemistry analysis of MFAP2 expression in gastric cancer samples. Data were obtained from the Human Protein Atlas. (A-E) Protein levels of MFAP2 in normal tissue (staining: not detected; intensity: negative; quantity: negative). (F-J) Levels of MFAP2 in tumor tissue (staining: not detected; intensity: negative; quantity: negative). Scale bar, $200 \mu \mathrm{m}$. MFAP2, microfibril-associated protein 2. Normal tissue $(\mathrm{n}=5)$, tumor tissue $(\mathrm{n}=12)$. 
promote epithelial-mesenchymal transition via activating the TGF- $\beta /$ SMAD2/3 signaling pathway in GC cells. Consistent with previous findings, our results suggested that MFAP2 may play a crucial role in progression of GC and that it may act as an oncogene. However, the functions and underlying mechanisms of MFAP2 involved in the development and progression of GC need to be further investigated.

To the best of our knowledge, no studies have been conducted evaluating the role of SLC5A6 in cancer to date. SLC5A6 encodes the $\mathrm{Na}^{+} /$multivitamin transporter (SMVT) (36), a member of the SLC5 family of $\mathrm{Na}^{+} /$solute symporters (37), and it mediates the $\mathrm{Na}^{+}$-dependent uptake of structurally diverse water-soluble vitamins, such as pantothenic acid and biotin $(38,39)$. Ghosal et al $(40)$ generated an intestine-specific (conditional) SMVT knockout (KO) mouse model using Cre/lox technology. They found that the KO mice exhibited growth retardation, decreased bone density, decreased bone length and decreased biotin status, and about two-thirds of the $\mathrm{KO}$ mice died prematurely between the age of 6 and 10 weeks (40). Our results indicated that SLC5A6 was a potential diagnostic and prognostic biomarker for GC, which implied that SLC5A6 was likely to play a role in tumorigenesis and progression. Details need to be clarified in further studies.

Other prognostic biomarkers for GC were previously identified via WGCNA using different GEO datasets, including sorting nexin 10 (41), elastin microfibril interface 1 (42) and follistatin like 1 (42). The present study has various limitations. First, the expression levels of SLC5A6 and MFAP2 were not further analyzed in clinical specimens or GC cell lines. Second, the functions and molecular regulatory mechanisms of SLC5A6 and MFAP2 in GC need to be further explored. Third, in this study, the sample size for screening hub genes was limited and further studies could include a lager sample pool to validate the results and conclusions presented here.

In conclusion, WGCNA was utilized to identify hub genes in GC that were further studied using RNA sequencing and available clinical data from TCGA. SLC5A6 and MFAP2 were hub genes associated oncogenesis and may act as independent prognostic factors for OS in GC patients. SLC5A6 and MFAP2 further may have the potential to be diagnostic and prognostic biomarkers in GC patients contributing to personalized therapy. However, further in-depth investigations are required to clarify the clinical and biological functions of these candidates.

\section{Acknowledgements}

We would like to thank Dr Xiuzhi Duan (The Second Affiliated Hospital of Zhejiang University School of Medicine) for constructive suggestions and supporting this study.

\section{Funding}

This study was supported by the National Natural Science Foundation of China Youth Science Foundation Project (grant no. 81802571), the National Natural Science Foundation of China (grant no. 81271917), and the Zhejiang Medical, Health Science and Technology Project (grant no. 2019RC039).

\section{Availability of data and materials}

All data generated or analyzed during this study are included either in this article or in the supplementary information.

\section{Authors' contributions}

WL, ZT and TS designed the study. WL, TS, DW, YS, ZL, YW, YD, PY and XD analyzed the data and prepared figures and/or tables. WL and ZT prepared the manuscript. All authors read and approved the final version of the manuscript.

\section{Ethics approval and consent to participate}

Not applicable.

\section{Patient consent for publication}

Not applicable.

\section{Competing interests}

The authors declare that they have no competing interests.

\section{References}

1. Van Cutsem E, Sagaert X, Topal B, Haustermans K and Prenen H: Gastric cancer. Lancet 388: 2654-2664, 2016.

2. Chmiela M, Karwowska Z, Gonciarz W, Allushi B and Stączek P: Host pathogen interactions in helicobacter pylori related gastric cancer. World J Gastroenterol 23: 1521-1540, 2017.

3. Zhu Q, Wong AK, Krishnan A, Aure MR, Tadych A, Zhang R, Corney DC, Greene CS, Bongo LA, Kristensen VN, et al: Targeted exploration and analysis of large cross-platform human transcriptomic compendia. Nat Methods 12: 211-214, 2015.

4. Alberti C, Manzenreither RA, Sowemimo I, Burkard TR, Wang J, Mahofsky K, Ameres SL and Cochella L: Cell-type specific sequencing of microRNAs from complex animal tissues. Nat Methods 15: 283-289, 2018.

5. Wu J, Xu J, Liu B, Yao G, Wang P, Lin Z, Huang B, Wang X, Li T, Shi S, et al: Chromatin analysis in human early development reveals epigenetic transition during ZGA. Nature 557: 256-260, 2018.

6. Rodriguez R and Miller KM: Unravelling the genomic targets of small molecules using high-throughput sequencing. Nat Rev Genet 15: 783-796, 2014.

7. Schwartz R and Schäffer AA: The evolution of tumour phylogenetics: Principles and practice. Nat Rev Genet 18: 213-229, 2017.

8. Simon R and Roychowdhury S: Implementing personalized cancer genomics in clinical trials. Nat Rev Drug Discov 12: 358-369, 2013.

9. Cancer Genome Atlas Research Network, Analysis Working Group, Asan University, BC Cancer Agency, Brigham and Women's Hospital, Broad Institute, Brown University, Case Western Reserve University, Dana-Farber Cancer Institute, Duke University, et al: Integrated genomic characterization of oesophageal carcinoma. Nature 541: 169-175, 2017.

10. Cancer Genome Atlas Research Network, Weinstein JN, Collisson EA, Mills GB, Shaw KR, Ozenberger BA, Ellrott K, Shmulevich I, Sander C and Stuart JM: The cancer genome atlas pan-cancer analysis project. Nat Genet 45: 1113-1120, 2013.

11. Zhu Y, Qiu P and Ji Y: TCGA-assembler: Open-source software for retrieving and processing TCGA data. Nat Methods 11: 599-600, 2014.

12. Uhlen M, Zhang C, Lee S, Sjöstedt E, Fagerberg L, Bidkhori G, Benfeitas R, Arif M, Liu Z, Edfors F, et al: A pathology atlas of the human cancer transcriptome. Science 357: eaan2507, 2017.

13. Baughman JM, Perocchi F, Girgis HS, Plovanich M, Belcher-Timme CA, Sancak Y, Bao XR, Strittmatter L, Goldberger O, Bogorad RL, et al: Integrative genomics identifies $\mathrm{MCU}$ as an essential component of the mitochondrial calcium uniporter. Nature 476: 341-345, 2011. 
14. Radulescu E, Jaffe AE, Straub RE, Chen Q, Shin JH, Hyde TM, Kleinman JE and Weinberger DR: Identification and prioritization of gene sets associated with schizophrenia risk by co-expression network analysis in human brain. Mol Psychiatry 2018 (Epub ahead of print).

15. Magani F, Bray ER, Martinez MJ, Zhao N, Copello VA, Heidman L, Peacock SO, Wiley DJ, D'Urso G and Burnstein KL: Identification of an oncogenic network with prognostic and therapeutic value in prostate cancer. Mol Syst Biol 14: e8202, 2018.

16. Liu W, Li L and Li W: Gene co-expression analysis identifies common modules related to prognosis and drug resistance in cancer cell lines. Int J Cancer 135: 2795-2803, 2014.

17. Wu H, Chen S, Yu J, Li Y, Zhang XY, Yang L, Zhang H, Hou Q, Jiang M, Brunicardi FC, et al: Single-cell transcriptome analyses reveal molecular signals to intrinsic and acquired paclitaxel resistance in esophageal squamous cancer cells. Cancer Lett 420: 156-167, 2018.

18. Punt S, Houwing-Duistermaat JJ, Schulkens IA, Thijssen VL, Osse EM, de Kroon CD, Griffioen AW, Fleuren GJ, Gorter A and Jordanova ES: Correlations between immune response and vascularization qRT-PCR gene expression clusters in squamous cervical cancer. Mol Cancer 14: 71, 2015.

19. Pasini FS, Zilberstein B, Snitcovsky I, Roela RA, Mangone FR, Ribeiro U Jr, Nonogaki S, Brito GC, Callegari GD, Cecconello I, et al: A gene expression profile related to immune dampening in the tumor microenvironment is associated with poor prognosis in gastric adenocarcinoma. J Gastroenterol 49: 1453-1466, 2014

20. American Joint Committee on Cancer: AJCC Cancer Staging Manual. 7th edition. Springer, New York, NY, 2017.

21. Tang J, Kong D, Cui Q, Wang K, Zhang D, Gong Y and Wu G: Prognostic genes of breast cancer identified by gene co-expression network analysis. Front Oncol 8: 374, 2018.

22. Xiao J and Yiqing Q: Bioinformatics analysis of the gene expression profile in bladder carcinoma. Genet Mol Biol 36: 287-291, 2013.

23. Zhang X, Li M, Chen S, Hu J, Guo Q, Liu R, Zheng H, Jin Z, Yuan Y, Xi Y and Hua B: Endoscopic screening in Asian countries is associated with reduced gastric cancer mortality: A meta-analysis and systematic review. Gastroenterology 155: 347-354.e9, 2018.

24. Tan P and Yeoh KG: Genetics and molecular pathogenesis of gastric adenocarcinoma. Gastroenterology 149: 1153-1162.e3, 2015.

25. Byron SA, Van Keuren-Jensen KR, Engelthaler DM, Carpten JD and Craig DW: Translating RNA sequencing into clinical diagnostics: Opportunities and challenges. Nat Rev Genet 17: 257-271, 2016

26. Lei Z, Chai N, Tian M, Zhang Y, Wang G, Liu J, Tian Z, Yi X, Chen D, Li X, et al: Novel peptide GX1 inhibits angiogenesis by specifically binding to transglutaminase- 2 in the tumorous endothelial cells of gastric cancer. Cell Death Dis 9: 579, 2018.

27. Tokumoto MW, Tanaka H, Tauchi Y, Kasashima H, Kurata K, Yashiro M, Sakurai K, Toyokawa T, Kubo N, Amano R, et al: Identification of tumour-reactive lymphatic endothelial cells capable of inducing progression of gastric cancer. Br J Cancer 113: 1046-1054, 2015.

28. Bae IH, Park MJ, Yoon SH, Kang SW, Lee SS, Choi KM and Um HD: Bcl-w promotes gastric cancer cell invasion by inducing matrix metalloproteinase-2 expression via phosphoinositide 3-kinase, Akt, and Sp1. Cancer Res 66: 4991-4995, 2006.
29. Kim LC, Cook RS and Chen J: mTORC1 and mTORC2 in cancer and the tumor microenvironment. Oncogene 36: 2191-2201, 2017.

30. Varn FS, Wang Y, Mullins DW, Fiering S and Cheng C: Systematic pan-cancer analysis reveals immune cell interactions in the tumor microenvironment. Cancer Res 77: 1271-1282, 2017.

31. Clarke AW and Weiss AS: Microfibril-associated glycoprotein-1 binding to tropoelastin: Multiple binding sites and the role of divalent cations. Eur J Biochem 271: 3085-3090, 2004.

32. Tatano Y, Takahashi T, Tsuji D, Takeuchi N, Tsuta K, Takada G, Ohsawa M, Sakuraba H and Itoh K: Significant decrease in tropoelastin gene expression in fibroblasts from a Japanese costello syndrome patient with impaired elastogenesis and enhanced proliferation. J Biochem 140: 193-200, 2006.

33. Zaravinos A, Kanellou P, Lambrou GI and Spandidos DA: Gene set enrichment analysis of the NF- $\kappa \mathrm{B} / \mathrm{Snail} / \mathrm{Y} Y 1 / \mathrm{RKIP}$ circuitry in multiple myeloma. Tumour Biol 35: 4987-5005, 2014

34. Silveira NJ, Varuzza L, Machado-Lima A, Lauretto MS, Pinheiro DG, Rodrigues RV, Severino P, Nobrega FG, Head and Neck Genome Project GENCAPO, Silva WA Jr, et al: Searching for molecular markers in head and neck squamous cell carcinomas (HNSCC) by statistical and bioinformatic analysis of larynx-derived SAGE libraries. BMC Med Genomics 1: 56, 2008.

35. Wang JK, Wang WJ, Cai HY, Du BB, Mai P, Zhang LJ, Ma W, $\mathrm{Hu}$ YG, Feng SF and Miao GY: MFAP2 promotes epithelial-mesenchymal transition in gastric cancer cells by activating TGF- $\beta /$ SMAD2/3 signaling pathway. Onco Targets Ther 11: 4001-4017, 2018

36. Hediger MA, Romero MF, Peng JB, Rolfs A, Takanaga H and Bruford EA: The ABCs of solute carriers: Physiological, pathological and therapeutic implications of human membrane transport proteins introduction. Pflugers Arch 447: 465-468, 2004.

37. Saier MH Jr: A functional-phylogenetic classification system for transmembrane solute transporters. Microbiol Mol Biol Rev 64: 354-411, 2000.

38. Subramanian VS, Constantinescu AR, Benke PJ and Said HM Mutations in SLC5A6 associated with brain, immune, bone, and intestinal dysfunction in a young child. Hum Genet 136: 253-261, 2017.

39. Vadlapudi AD, Vadlapatla RK and Mitra AK: Sodium dependent multivitamin transporter (SMVT): A potential target for drug delivery. Curr Drug Targets 13: 994-1003, 2012.

40. Ghosal A, Lambrecht N, Subramanya SB, Kapadia R and Said HM: Conditional knockout of the Slc5a6 gene in mouse intestine impairs biotin absorption. Am J Physiol Gastrointest Liver Physiol 304: G64-G71, 2013.

41. Zhang J, Wu Y, Jin HY, Guo S, Dong Z, Zheng ZC, Wang Y and Zhao Y: Prognostic value of sorting nexin 10 weak expression in stomach adenocarcinoma revealed by weighted gene co-expression network analysis. World J Gastroenterol 24: 4906-4919, 2018

42. Chen J, Wang X, Hu B, He Y, Qian X and Wang W: Candidate genes in gastric cancer identified by constructing a weighted gene co-expression network. PeerJ 6: e4692, 2018.

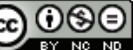

This work is licensed under a Creative Commons Attribution-NonCommercial-NoDerivatives 4.0 International (CC BY-NC-ND 4.0) License. 